\title{
Front Matter: Volume 6914
}

, "Front Matter: Volume 6914," Proc. SPIE 6914, Medical Imaging 2008: Image Processing, 691401 (28 March 2008); doi: 10.1117/12.798638

SPIE. Event: Medical Imaging, 2008, San Diego, California, United States 


\title{
PROGRESS IN BIOMEDICAL OPTICS AND IMAGING
}

Vol. 9, No. 31

\section{Medical Imaging 2008}

Image Processing

\author{
Joseph M. Reinhardt \\ Josien P. W. Pluim \\ Editors
}

17-19 February 2008

San Diego, California, USA

Sponsored by

SPIE

Cooperating Organizations

AAPM-American Association of Physicists in Medicine (USA)

APS-American Physiological Society (USA)

CARS - Computer Assisted Radiology and Surgery (Germany)

IS\&T-The Society for Imaging Science and Technology

MIPS-Medical Image Perception Society (USA)

RSNA-Radiological Society of North America (USA)

SIIM-Society for Imaging Informatics in Medicine (USA)

SMI-The Society for Molecular Imaging

The DICOM Standards Committee (USA)

Published by

SPIE

Part One of Three Parts

Volume 6914 
The papers included in this volume were part of the technical conference cited on the cover and title page. Papers were selected and subject to review by the editors and conference program committee. Some conference presentations may not be available for publication. The papers published in these proceedings reflect the work and thoughts of the authors and are published herein as submitted. The publisher is not responsible for the validity of the information or for any outcomes resulting from reliance thereon.

Please use the following format to cite material from this book:

Author(s), "Title of Paper," in Medical Imaging 2008: Image Processing, edited by Joseph M. Reinhardt, Josien P. W. Pluim, Proceedings of SPIE Vol. 6914 (SPIE, Bellingham, WA, 2008) Article CID Number.

ISSN 1605-7422

ISBN 9780819470980

Published by

SPIE

P.O. Box 10, Bellingham, Washington 98227-0010 USA

Telephone +1 3606763290 (Pacific Time) · Fax +1 3606471445

SPIE.org

Copyright (C) 2008, Society of Photo-Optical Instrumentation Engineers.

Copying of material in this book for internal or personal use, or for the internal or personal use of specific clients, beyond the fair use provisions granted by the U.S. Copyright Law is authorized by SPIE subject to payment of copying fees. The Transactional Reporting Service base fee for this volume is $\$ 18.00$ per article (or portion thereof), which should be paid directly to the Copyright Clearance Center (CCC), 222 Rosewood Drive, Danvers, MA 01923. Payment may also be made electronically through CCC Online at copyright.com. Other copying for republication, resale, advertising or promotion, or any form of systematic or multiple reproduction of any material in this book is prohibited except with permission in writing from the publisher. The CCC fee code is 1605 $7422 / 08 / \$ 18.00$.

Printed in the United States of America.

Publication of record for individual papers is online in the SPIE Digital Library.

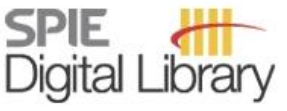

SPIEDigitallibrary.org

Paper Numbering: Proceedings of SPIE follow an e-First publication model, with papers published first online and then in print and on CD-ROM. Papers are published as they are submitted and meet publication criteria. A unique, consistent, permanent citation identifier (CID) number is assigned to each article at the time of the first publication. Utilization of CIDs allows articles to be fully citable as soon they are published online, and connects the same identifier to all online, print, and electronic versions of the publication. SPIE uses a six-digit CID article numbering system in which:

- The first four digits correspond to the SPIE volume number.

- The last two digits indicate publication order within the volume using a Base 36 numbering system employing both numerals and letters. These two-number sets start with 00, 01, 02, 03, 04, $05,06,07,08,09,0 A, 0 B \ldots$. 0Z, followed by 10-1Z, 20-2Z, etc.

The CID number appears on each page of the manuscript. The complete citation is used on the first page, and an abbreviated version on subsequent pages. Numbers in the index correspond to the last two digits of the six-digit CID number. 


\title{
Contents
}

\author{
xxi Conference Committee \\ xxiii Introduction \\ xxv In Memoriam: Sam Dwyer (1932-2008)
}

\section{Part One}

\section{SESSION 1 SEGMENTATION I: METHODOLOGY}

691402 Area prior constrained level set evolution for medical image segmentation [6914-01] I. Ben Ayed, S. Li, GE Healthcare (Canada); A. Islam, G. Garvin, St. Joseph's Health Care (Canada); R. Chhem, London Health Sciences Ctr. (Canada)

691403 A resistive-network model for image segmentation [6914-02] P. J. Yim, UMDNJ-Robert Wood Johnson Medical School (USA)

691404 A new distribution metric for image segmentation [6914-03] R. Sandhu, Georgia Institute of Technology (USA); T. Georgiou, Univ. of Minnesota (USA); A. Tannenbaum, Georgia Institute of Technology (USA)

691405 Segmenting images analytically in shape space [6914-04] Y. Rathi, Brigham and Women's Hospital, Harvard Medical School (USA); S. Dambreville, Georgia Institute of Technology (USA); M. Niethammer, Brigham and Women's Hospital, Harvard Medical School (USA); J. Malcolm, Georgia Institute of Technology (USA); J. Levitt, M. E. Shenton, Brigham and Women's Hospital, Harvard Medical School (USA);

A. Tannenbaum, Georgia Institute of Technology (USA)

691406 A unified framework for joint registration and segmentation [6914-05]

K. Ens, Univ. of Lübeck (Germany) and Philips Research Europe - Hamburg (Germany); J. von Berg, S. Kabus, C. Lorenz, Philips Research Europe - Hamburg (Germany); B. Fischer, Univ. of Lübeck (Germany)

\section{SESSION $2 \quad$ ATLASES AND POPULATION STUDIES}

691407 Adaptive local multi-atlas segmentation: application to heart segmentation in chest CT scans [6914-06]

E. M. van Rikxoort, I. Isgum, M. Staring, S. Klein, B. van Ginneken, Univ. Medical Ctr. Utrecht (Netherlands)

691408 Robust registration between cardiac MRI images and atlas for segmentation propagation [6914-07]

X. Zhuang, D. J. Hawkes, W. R. Crum, Univ. College London (United Kingdom);

R. Boubertakh, S. Uribe, King's College London (United Kingdom); D. Atkinson, Univ. College London (United Kingdom); P. Batchelor, T. Schaeffter, R. Razavi, King's College London (United Kingdom); D. L. G. Hill, Univ. College London (United Kingdom) 
691409 The SRI24 multichannel brain atlas: construction and applications [6914-08]

T. Rohlfing, SRI International (USA); N. M. Zahr, SRI International (USA) and Stanford Univ. (USA); E. V. Sullivan, Stanford Univ. (USA); A. Pfefferbaum, SRI International (USA) and Stanford Univ. (USA)

6914 OA A generalization of voxel-wise procedures for high-dimensional statistical inference using ridge regression [6914-09]

K. Sjöstrand, EXINI Diagnostics AB (Sweden); V. A. Cardenas, Univ. of California, San Francisco (USA); R. Larsen, Technical Univ. of Denmark (Denmark); C. Studholme, Univ. of California, San Francisco (USA)

$6914 \mathrm{OB}$ The evaluation of a population based diffusion tensor image atlas using a ground truth method [6914-10]

W. Van Hecke, Univ. of Antwerp (Belgium); A. Leemans, Cardiff Univ. (United Kingdom) and Univ. of Antwerp (Belgium); E. D'Agostino, Katholieke Univ. Leuven (Belgium); S. De Backer, E. Vandervliet, P. M. Parizel, J. Sijbers, Univ. of Antwerp (Belgium)

6914 OC Multivariate longitudinal statistics for neonatal-pediatric brain tissue development [6914-11] S. XU, M. Styner, J. Gilmore, Univ. of North Carolina, Chapel Hill (USA); G. Gerig, Univ. of Utah (USA)

\section{SESSION 3 REGISTRATION I: APPLICATIONS}

6914 OE Mosaicing of single plane illumination microscopy images using groupwise registration and fast content-based image fusion [6914-13]

S. Preibisch, Max Planck Institute of Molecular Cell Biology and Genetics (Germany); T. Rohlfing, M. P. Hasak, SRI International (USA); P. Tomancak, Max Planck Institute of Molecular Cell Biology and Genetics (Germany)

6914 OF Ultrasound specific similarity measures for three-dimensional mosaicing [6914-14] C. Wachinger, N. Navab, Technische Univ. München (Germany)

6914 OG Three-dimensional image registration of MR proximal femur images for the analysis of trabecular bone parameters [6914-15]

J. Blumenfeld, Univ. of California, San Francisco (USA) and UCSF-UCB Joint Graduate Group in Bioengineering (USA); C. Studholme, J. Carballido-Gamio, T. M. Link, S. Majumdar, Univ. of California, San Francisco (USA)

$6914 \mathrm{OH}$ Vertebral surface registration using ridgelines/crestlines [6914-16]

S. Tan, National Institute of Arthritis and Musculoskeletal and Skin Diseases (USA); J. Yao, L. Yao, R. M. Summers, National Institutes of Health (USA); M. M. Ward, National Institute of Arthritis and Musculoskeletal and Skin Diseases (USA)

6914 Ol Bi-planar 2D-to-3D registration in Fourier domain for stereoscopic x-ray motion tracking [6914-17]

D. Zosso, B. Le Callennec, M. Bach Cuadra, K. Aminian, Ecole Polytechnique Fédérale de Lausanne (Switzerland); B. M. Jolles, Hôpital Orthopédique de la Suisse Romande and University Hospital of Lausanne (Switzerland); J.-P. Thiran, Ecole Polyłechnique Fédérale de Lausanne (Switzerland) 
$69140 \mathrm{~J}$ Cortical thickness measurement from magnetic resonance images using partial volume estimation [6914-18]

M. A. Zuluaga, ICT Ctr., CSIRO (Australia) and Univ. de los Andes (Colombia); O. Acosta, P. Bourgeat, ICT Ctr., CSIRO (Australia); M. Hernández Hoyos, Univ. de Los Andes (Colombia); O. Salvado, ICT Ctr., CSIRO (Australia); S. Ourselin, ICT Ctr., CSIRO (Australia) and Univ. College London (United Kingdom)

6914 OK Parallel optimization of tumor model parameters for fast registration of brain tumor images [6914-19]

E. I. Zacharaki, C. S. Hogea, D. Shen, G. Biros, C. Davatzikos, Univ. of Pennsylvania (USA)

$6914 \mathrm{OL}$ Spatial normalization of diffusion tensor images based on anisotropic segmentation [6914-20]

J. Yang, D. Shen, C. Misra, X. Wu, Univ. of Pennsylvania (USA); S. Resnick, National Institute on Aging (USA); C. Davatzikos, R. Verma, Univ. of Pennsylvania (USA)

6914 OM Fusion of rat brain histology and MRI using weighted multi-image mutual information [6914-21]

C. Palm, Univ. College London (United Kingdom) and Research Ctr. Jülich (Germany);

G. P. Penney, W. R. Crum, King's College London (United Kingdom); J. A. Schnabel, Univ. of Oxford (United Kingdom); U. Pietrzyk, Research Ctr. Jülich (Germany) and Univ. Wuppertal (Germany); D. J. Hawkes, Univ. College London (United Kingdom)

6914 ON Comparison of EM-based and level set partial volume segmentations of MR brain images [6914-22]

H. D. Tagare, Yale Univ. (USA); Y. Chen, Univ. of Florida (USA); R. K. Fulbright, Yale Univ. (USA)

691400 3D MRI brain image segmentation based on region restricted EM algorithm [6914-23]

Z. Li, J. Fan, The Univ. of North Carolina at Charlotte (USA)

6914 OP Automatic segmentation of the facial nerve and chorda tympani using image registration and statistical priors [6914-24]

J. H. Noble, Vanderbilt Univ. (USA); F. M. Warren, Univ. of Utah (USA); R. F. Labadie,

Vanderbilt Univ. Medical Ctr. (USA); B. M. Dawant, Vanderbilt Univ. (USA)

\section{SESSION 5 CLASSIFICATION AND PATTERN RECOGNITION}

$69140 Q \quad$ Feature selection and classification of multiparametric medical images using bagging and SVM [6914-25]

Y. Fan, Univ. of Pennsylvania (USA); S. M. Resnick, National Institute on Aging (USA);

C. Davatzikos, Univ. of Pennsylvania (USA)

6914 OR Bleeding detection in wireless capsule endoscopy using adaptive colour histogram model and support vector classification [6914-26]

M. W. Mackiewicz, M. Fisher, Univ. of East Anglia (United Kingdom); C. Jamieson, Norfolk \& Norwich Univ. Hospital (United Kingdom) 
6914 OS Statistical modeling and MAP estimation for body fat quantification with MRI ratio imaging [6914-27]

W. C. K. Wong, D. H. Johnson, Case Western Reserve Univ. (USA); D. L. Wilson, Case Western Reserve Univ. (USA) and University Hospitals of Cleveland (USA)

6914 OT A variational method for automatic localization of the most pathological ROI in the knee cartilage [6914-28]

A. A. Qazi, Univ. of Copenhagen (Denmark); E. B. Dam, Nordic Bioscience Imaging (Denmark); M. Loog, M. Nielsen, Univ. of Copenhagen (Denmark) and Nordic Bioscience Imaging (Denmark); F. Lauze, Nordic Bioscience Imaging (Denmark); C. Christiansen, Ctr. for Clinical and Basic Research (Denmark)

6914 OU Motion blur detection in radiographs [6914-29]

H. Luo, W. J. Sehnert, J. S. Ellinwood, D. Foos, Carestream Health, Inc. (USA); B. Reiner,

E. Siegel, Univ. of Maryland (USA)

\section{SESSION 6 REGISTRATION II: METHODOLOGY}

$69140 \mathrm{~V}$ Hybrid physics-based elastic image registration using approximating splines [6914-30] S. Wörz, K. Rohr, Univ. of Heidelberg (Germany) and German Cancer Research Ctr. (Germany)

6914 OW On the development of a new non-rigid image registration using deformation based grid generation [6914-31]

C.-Y. Hsieh, H. Chen, T.-H. Lin, H.-Y. Hsiao, M.-Y. Chu, G. Liao, The Univ. of Texas at Arlington (USA); H. Zhong, Virginia Commonwealth Univ. (USA)

$69140 X$ A novel framework for multi-modal intensity-based similarity measures based on internal similarity [6914-32]

G. P. Penney, King's College London (United Kingdom); L. D. Griffin, Univ. College London (United Kingdom); A. P. King, King's College London (United Kingdom); D. J. Hawkes, Univ. College London (United Kingdom)

6914 OY Volume preserving image registration via a post-processing stage [6914-33]

R. Hameeteman, J. F. Veenland, University Medical Ctr. Rotterdam (Netherlands); W. J. Niessen, University Medical Ctr. Rotterdam (Netherlands) and Delft Univ. of Technology (Netherlands)

$69140 Z$ Improved CT and MR image registration with the introduction of a dual-modality contrast agent: performance assessment using quantitative and information theoretic methods [6914-34]

J. D. P. Hoisak, J. Zheng, Univ. of Toronto (Canada) and Princess Margaret Hospital, University Health Network (Canada); C. Allen, Univ. of Toronto (Canada); D. A. Jaffray, Univ. of Toronto (Canada) and Princess Margaret Hospital, University Health Network (Canada)

691410 Conditional statistical model building [6914-35]

M. F. Hansen, M. S. Hansen, R. Larsen, Technical Univ. of Denmark (Denmark) 
$691411 \quad$ Nonrigid registration of carotid ultrasound and MR images using a "twisting and bending" model [6914-36]

N. D. Nanayakkara, B. Chiu, Robarts Research Institute (Canada) and The Univ. of Western Ontario (Canada); A. Samani, The Univ. of Western Ontario (Canada); J. D. Spence, G. Parraga, Robarts Research Institute (Canada); J. Samarabandu, The Univ. Of Western Ontario (Canada); A. Fenster, Robarts Research Institute (Canada) and The Univ. of Western Ontario (Canada)

691412 Deformable registration of 3D vessel structures to a single projection image [6914-37] D. Zikic, M. Groher, Technische Univ. München (Germany); A. Khamene, Siemens Corporate Research (USA); N. Navab, Technische Univ. München (Germany)

691413 3D inters-subject cardiac registration using 4D information [6914-38] A. Lopez, Software Competence Ctr. Hagenberg (Austria) and Univ. for Health Sciences, Medical Informatics and Technology (Austria); K. D. Fritscher, Univ. for Health Sciences, Medical Informatics and Technology (Austria); T. Trieb, Innsbruck Medical Univ. (Austria); R. Schubert, Univ. for Health Sciences, Medical Informatics and Technology (Austria); J. Mattes, Software Competence Ctr. Hagenberg (Austria)

691414 Level set segmentation of the heart from 4D phase contrast MRI [6914-39] D. Kainmüller, R. Unterhinninghofen, Univ. of Karlsruhe (Germany); S. Ley, Germany Cancer Research Ctr. (Germany); R. Dillmann, Univ. of Karlsruhe (Germany)

691415 Segmentation of myocardial perfusion MR sequences with multi-band active appearance models driven by spatial and temporal features [6914-40]

N. Baka, Leiden Univ. Medical Ctr. (Netherlands), Delft Univ. of Technology (Netherlands), and Erasmus Medical Ctr. (Netherlands); J. Milles, Leiden Univ. Medical Ctr. (Netherlands); E. A. Hendriks, Leiden Univ. Medical Ctr. (Netherlands) and Delft Univ. of Technology (Netherlands); A. Suinesiaputra, Leiden Univ. Medical Ctr. (Netherlands); M. Jerosch Herold, Brigham and Women's Hospital (USA); J. H. C. Reiber, Leiden Univ. Medical Ctr. (Netherlands); B. P. F. Lelieveldt, Leiden Univ. Medical Ctr. (Netherlands) and Delft Univ. of Technology (Netherlands)

691416 Four-chamber heart modeling and automatic segmentation for 3D cardiac CT volumes [6914-41]

Y. Zheng, B. Georgescu, Siemens Corporate Research (USA); A. Barbu, Florida State Univ. (USA); M. Schevering, Siemens Medical Solutions (Germany); D. Comaniciu, Siemens Corporate Research (USA)

691417 Segmentation of the heart and major vascular structures in cardiovascular CT images [6914-42] J. Peters, O. Ecabert, Philips Research Europe - Aachen (Germany); C. Lorenz, J. von Berg, Philips Research Europe - Hamburg (Germany); M. J. Walker, T. B. Ivanc, M. Vembar, M. E. Olszewski, Philips Healthcare, CT Clinical Science (USA); J. Weese, Philips Research Europe - Aachen (Germany)

691418 AdaBoost classification for model-based segmentation of the outer wall of the common carotid artery in CTA [6914-135]

D. Vukadinovic, T. van Walsum, R. Manniesing, A. van der Lugt, T. T. de Weert, W. J. Niessen, University Medical Ctr. Rotterdam (Netherlands) 
691419 Image intensity standardization in 3D rotational angiography and its application to vascular segmentation [6914-44]

H. Bogunović;, A. G. Radaelli, M. De Craene, D. Delgado, A. F. Frangi, Univ. Pompeu Fabra (Spain) and CIBER-BNN (Spain)

6914 1A Fast bias field reduction by localized Lloyd-Max quantization [6914-45]

R. Hanel, Medical Univ. of Vienna (Austria) and Univ. of Antwerp (Belgium);

K. J. Batenburg, S. De Backer, P. Scheunders, J. Sijbers, Univ. of Antwerp (Belgium)

6914 1B Feature-preserving artifact removal from dermoscopy images [6914-46]

H. Zhou, Georgia Institute of Technology (USA); M. Chen, R. Gass, Intel Research Pittsburgh (USA); J. M. Rehg, Georgia Institute of Technology (USA); L. Ferris, J. Ho, L. Drogowski, Univ. of Pittsburgh Medical Ctr. (USA)

6914 1C A quantitative performance measure for a clinical evaluation of comb structure removal algorithms in flexible endoscopy [6914-47]

S. Rupp, Fraunhofer-Institute for Integrated Circuits (Germany)

6914 1D Digital mammogram enhancement based on ROI enhancement and background suppression [6914-48]

R. Peng, P. K. Varshney, H. Chen, Syracuse Univ. (USA); J. H. Michels, JHM Technologies, LLC (USA)

\section{SESSION 9 LIVER APPLICATIONS}

$69141 \mathrm{~F}$ Adaptive directional region growing segmentation of the hepatic vasculature [6914-50] Q. Shang, L. Clements, R. L. Galloway, Vanderbilt Univ. (USA); W. C. Chapman, Washington Univ. in St. Louis (USA); B. M. Dawant, Vanderbilt Univ. (USA)

6914 IG Quantitative growth measurement of lesions in hepatic interval CT exams [6914-51] S. Sarkar, R. Narayanan, H. Park, B. Ma, P. H. Bland, C. R. Meyer, Univ. of Michigan (USA)

$69141 \mathrm{H}$ Liver segmentation combining Gabor filtering and traditional vector field snake [6914-52] A. M. Mintz, Carnegie-Mellon Univ. (USA); D. S. Raicu, J. D. Furst, DePaul Univ. (USA)

\section{SESSION 10 PULMONARY APPLICATIONS}

$691411 \quad$ Lung lobe modeling and segmentation with individualized surface meshes [6914-53] T. Blaffert, H. Barschdorf, J. von Berg, S. Dries, A. Franz, T. Klinder, C. Lorenz, S. Renisch, R. Wiemker, Philips Research Europe - Hamburg (Germany)

$69141 \mathrm{~J} \quad$ Robust system for human airway-tree segmentation [6914-54] M. W. Graham, J. D. Gibbs, W. E. Higgins, The Pennsylvania State Univ. (USA)

$69141 \mathrm{~K} \quad$ Voxel classification based airway tree segmentation [6914-55] P. Lo, Univ. of Copenhagen (Denmark); M. de Bruijne, Univ. of Copenhagen (Denmark) and University Medical Ctr. Rotterdam (Netherlands) 
6914 1L 4DCT image-based lung motion field extraction and analysis [6914-56]

T. Klinder, Leibniz Univ. Hannover (Germany) and Philips Research Europe - Hamburg

(Germany); C. Lorenz, J. von Berg, S. Renisch, T. Blaffert, Philips Research Europe - Hamburg

(Germany); J. Ostermann, Leibniz Univ. Hannover (Germany)

$69141 \mathrm{M}$ The evaluation of a highly automated mixture model based technique for PET fumor volume segmentation [6914-57]

M. Aristophanous, C. A. Pelizzari, The Univ. of Chicago (USA)

\section{Part Two}

\section{SESSION 11 SEGMENTATION II: APPLICATIONS}

$69141 \mathrm{~N}$ Shape priors for segmentation of the cervix region within uterine cervix images [6914-58]

S. Lotenberg, S. Gordon, H. Greenspan, Tel Aviv Univ. (Israel)

691410 Use of a CT statistical deformation model for multi-modal pelvic bone segmentation [6914-59]

S. Thompson, Univ. College London (United Kingdom); G. Penney, King's College London (United Kingdom); D. Buie, Univ. College London (United Kingdom); P. Dasgupta, Guy's and

St Thomas' Hospital (United Kingdom); D. Hawkes, Univ. College London (United Kingdom)

$69141 \mathrm{P}$ Prostate segmentation from 3D transrectal ultrasound using statistical shape models and various appearance models [6914-60]

T. Heimann, M. Baumhaver, T. Simpfendörfer, H.-P. Meinzer, I. Wolf, German Cancer Research Ctr. (Germany)

$69141 Q \quad$ Fuzzy pulmonary vessel segmentation in contrast enhanced CT data [6914-118] J. N. Kaftan, RWTH Aachen Univ. (Germany) and Siemens Medical Solutions (Germany); A. P. Kiraly, Siemens Corporate Research (USA); A. Bakai, Siemens Medical Solutions (Germany); M. Das, RWTH Aachen Univ. (Germany); C. L. Novak, Siemens Corporate Research (USA); T. Aach, RWTH Aachen Univ. (Germany)

$69141 R \quad V e s s e l$ segmentation in 3D spectral OCT scans of the retina [6914-62] M. Niemeijer, M. K. Garvin, The Univ. of lowa (USA); B. van Ginneken, University Medical Ctr. Utrecht (Netherlands); M. Sonka, M. D. Abràmoff, The Univ. of lowa (USA)

6914 iS Lymph node segmentation on CT images by a shape model guided deformable surface method [6914-63]

D. Maleike, German Cancer Research Ctr. (Germany); M. Fabel, Univ. Hospital SchleswigHolstein (Germany); R. Tetzlaff, H. von Tengg-Kobligk, T. Heimann, H.-P. Meinzer, I. Wolf, German Cancer Research Ctr. (Germany)

6914 IT A novel shape prior based segmentation of touching or overlapping ellipse-like nuclei [6914-64]

X. He, Q. Liao, Tsinghua Univ. (China) 
$69141 \mathrm{U}$ A machine learning approach for body part recognition based on CT images [6914-65] K. Nakamura, Y. Li, W. Ito, K. Shimura, FUJIFILM Corp. (Japan)

$69141 \mathrm{~V}$ Personal identification based on blood vessels of retinal fundus images [6914-66] K. Fukuta, T. Nakagawa, Gifu Univ. (Japan); Y. Hayashi, TAK Co., Ltd. (Japan); Y. Hatanaka, Gifu National College of Technology (Japan); T. Hara, H. Fujita, Gifu Univ. (Japan)

6914 IW Efficient classifier generation and weighted voting for atlas-based segmentation: two small steps faster and closer to the combination oracle (Cum Laude Poster Award) [6914-67] X. Artaechevarria, A. Muñoz-Barrutia, C. Ortiz-de-Solorzano, Univ. of Navarra (Spain)

$69141 \mathrm{X}$ Scene analysis with structural prototypes for content-based image retrieval in medicine [6914-68]

B. Fischer, M. Sauren, M. O. Güld, T. M. Deserno, RWTH Aachen Univ. of Technology (Germany)

6914 IY A co-occurrence texture semi-invariance to direction, distance, and patient size [6914-69] R. Susomboon, D. Raicu, J. Furst, DePaul Univ. (USA); T. B. Johnson, Northwestern Univ. (USA)

691412 Tissue classification using cluster features for lesion detection in digital cervigrams [6914-70]

X. Huang, W. Wang, Lehigh Univ. (USA); Z. Xue, S. Antani, L. R. Long, National Library of Medicine (USA); J. Jeronimo, National Cancer Institute (USA)

POSTERS: IMAGE RESTORATION AND ENHANCEMENT

691420 An image reconstruction method based on machine learning for dual-energy subtraction radiography [6914-71]

Y. Kitamura, M. Yamada, W. Ito, FUJIFILM Corp. (Japan)

691421 Development of adaptive noise reduction filter algorithm for pediatric body images in a multi-detector CT [6914-72]

E. Nishimaru, Hiroshima City Hospital (Japan) and Kanazawa Univ. (Japan); K. Ichikawa, Kanazawa Univ. (Japan); I. Okita, Y. Ninomiya, Y. Tomoshige, T. Kurokawa, Y. Ono, Hiroshima City Hospital (Japan); Y. Nakamura, Kure City Medical Association Hospital (Japan); M. Suzuki, Kanazawa Univ. (Japan)

691422 Retinal vessel enhancement based on directional field [6914-74]

J. Chen, J. Tian, Institute of Automation (China)

691423 Fast multiscale vessel enhancement filtering [6914-75]

D. H. Ye, D. Kwon, Seoul National Univ. (South Korea); I. D. Yun, Hankuk Univ. of Foreign Studies (South Korea); S. U. Lee, Seoul National Univ. (South Korea)

691424 Adaptive kernel algorithm for FPGA-based speckle reduction [6914-76]

G. Tech, R. Schwann, G. Kappen, M. Först, T. G. Noll, RWTH Aachen Univ. (Germany)

691425 Enhancing regional lymph nodes from endoscopic ultrasound images [6914-77]

I. Nwogu, V. Chaudhary, Univ. at Buffalo, SUNY (USA) 
691426 Clinical validation and performance evaluation of enhancement methods acquired from interventional C-ARM x-ray [6914-78]

L. Wei, D. Kumar, A. Khemka, Eigen LLC (USA); R. Turlapati, Univ. of Wisconsin (USA); J. S. Suri, Eigen LLC (USA)

691427 Dermascopic hair disocclusion using inpainting [6914-79]

P. Wighton, T. K. Lee, Simon Fraser Univ. (Canada), BC Cancer Research Ctr. (Canada), and Univ. of British Columbia and Vancouver Coastal Health Research Institute (Canada); M. S. Atkins, Simon Fraser Univ. (Canada)

691428 Denoising of brain MRI images using modified PDE model based on pixel similarity [6914-80]

R. Jin, Huazhong Univ. of Science and Technology (China); E. Song, Huazhong Univ. of Science and Technology (China) and Jiangxi College of Chinese Medicine (China); L. Zhang, Z. Min, X. Xu, Huazhong Univ. of Science and Technology (China); C.-C. Huang, Southern Polytech State Univ. (USA)

691429 Pyramidal flux in an anisotropic diffusion scheme for enhancing structures in 3D images [6914-81]

O. Acosta, H. Frimmel, ICT Ctr., CSIRO (Australia); A. Fenster, Robarts Research Institute (Canada); O. Salvado, ICT Ctr., CSIRO (Australia); S. Ourselin, ICT Ctr., CSIRO (Australia) and Univ. College London (United Kingdom)

$69142 \mathrm{~A}$ Informative frame detection from wireless capsule video endoscopic images [6914-82] M. K. Bashar, K. Mori, Y. Suenaga, T. Kitasaka, Nagoya Univ. (Japan); Y. Mekada, Chukyo Univ. (Japan) and Nagoya Univ. (Japan)

\section{POSTERS: MOTION ANALYSIS}

$69142 \mathrm{~B}$ Automated motion correction based on target tracking for dynamic nuclear medicine studies [6914-83]

X. Cao, T. Tetrault, F. Fahey, T. Treves, Children's Hospital Boston, Harvard Medical School (USA)

$69142 \mathrm{C} \quad$ Multi-object tracking of human spermatozoa [6914-84]

L. Sørensen, J. Østergaard, P. Johansen, Univ. of Copenhagen (Denmark); M. de Bruijne, Univ. of Copenhagen (Denmark) and Erasmus MC (Netherlands)

$69142 \mathrm{D}$ Tracking the hyoid bone in videofluoroscopic swallowing studies (Honorable Mention Poster Award)[6914-85]

P. M. Kellen, D. Becker, J. M. Reinhardt, D. van Daele, The Univ. of lowa (USA)

POSTERS: MRI

$69142 \mathrm{E}$ Towards user-independent DTI quantification [6914-86]

J. Klein, H. Stuke, J. Rexilius, MeVis Research (Germany); B. Stieltjes, German Cancer

Research Ctr. (Germany); H. K. Hahn, H.-O. Peitgen, MeVis Research (Germany) 
$69142 \mathrm{~F} \quad$ An exploration of spatial similarities in temporal noise spectra in fMRI measurements [6914-87]

D. H. J. Poot, J. Sijbers, Univ. of Antwerp (Belgium); A. J. den Dekker, Delft Univ. of Technology (Netherlands)

$69142 \mathrm{G}$ White matter tractographies registration using Gaussian mixture modeling [6914-89] O. Zvitia, A. Mayer, H. Greenspan, Tel Aviv Univ. (Israel)

$69142 \mathrm{H} \quad$ Tensor distribution function [6914-90]

A. D. Leow, S. Zhu, Univ. of California, Los Angeles (USA)

$691421 \quad$ Susceptibility correction for improved tractography using high field DT-EPI [6914-91] W. Pintjens, D. H. J. Poot, M. Verhoye, A. Van Der Linden, J. Sijbers, Univ. of Antwerp (Belgium)

$69142 \mathrm{~J} \quad$ A Bayesian method with reparameterization for diffusion tensor imaging [6914-92] D. Zhou, I. L. Dryden, A. Koloydenko, B. Li, Univ. of Nottingham (United Kingdom)

$69142 \mathrm{~K}$ Automatic regional analysis of DTI properties in the developmental macaque brain [6914-93]

M. Styner, R. Knickmeyer, Univ. of North Carolina, Chapel Hill (USA); C. Coe, S. J. Short, Univ. of Wisconsin, Madison (USA); J. Gilmore, Univ. of North Carolina, Chapel Hill (USA)

\section{POSTERS: MULTIRESOLUTION AND WAVELETS}

$69142 \mathrm{~L}$ Short basis functions for constant-variance interpolation [6914-94]

P. Thévenaz, T. Blu, M. Unser, École Polytechnique Fédérale de Lausanne (Switzerland)

$69142 \mathrm{M}$ Efficient random access high resolution region-of-interest (ROI) image retrieval using backward coding of wavelet trees (BCWT) [6914-95]

E. Corona, B. Nutter, S. Mitra, Texas Tech Univ. (USA); J. Guo, Beijing Institute of Technology (China); T. Karp, Texas Tech Univ. (USA)

\section{POSTERS: REGISTRATION}

$69142 \mathrm{~N}$ Semi-automatic matching of OCT and IVUS images for image fusion [6914-43] O. Pauly, G. Unal, G. Slabaugh, Siemens Corporate Research (USA); S. Carlier, Cardiovascular Research Foundation (USA); T. Fang, Siemens Corporate Research (USA)

691420 Nonlinear elastic model for image registration and soft tissue simulation based on piecewise St. Venant-Kirchhoff material approximation [6914-96]

E. Gladilin, R. Eils, German Cancer Research Ctr. (Germany)

$69142 \mathrm{P} \quad$ Validation and comparison of registration methods for free-breathing 4D lung CT [6914-97] T. Vik, S. Kabus, J. von Berg, Philips Research Europe - Hamburg (Germany); K. Ens, Philips Research Europe - Hamburg (Germany) and Univ. of Lübeck (Germany); S. Dries, Philips Research Europe - Hamburg (Germany); T. Klinder, Philips Research Europe - Hamburg (Germany) and Leibniz Univ. Hannover (Germany); C. Lorenz, Philips Research Europe Hamburg (Germany) 
$69142 R \quad$ Non-rigid registration of 2D manifolds in 3D Euclidian space [6914-99]

S. Darkner, Technical Univ. of Denmark (Denmark) and Eriksholm Research Ctr. (Oticon A/S) (Denmark); M. Vester-Christensen, R. R. Paulsen, R. Larsen, Technical Univ. of Denmark (Denmark)

$69142 S$ Model-to-image based 2D-3D registration of angiographic data [6914-100]

S. Mollus, Philips Research Europe (Germany); J. Lübke, University Medical Ctr. Freiburg (Germany); A. J. Walczuch, Philips Research Europe (Germany); H. Schumann, Univ. of Rostock (Germany); J. Weese, Philips Research Europe (Germany)

$69142 \mathrm{R}$ Robust registration for change detection [6914-101]

S. Darkner, Technical Univ. of Denmark (Denmark) and Eriksholm Research Ctr. (Denmark);

D. Witzner Hansen, R. R. Paulsen, R. Larsen, Technical Univ. of Denmark (Denmark)

$69142 \mathrm{U}$ Reconstruction and registration of multispectral x-ray images for reliable alignment correction in radiation treatment devices [6914-102]

B. P. Selby, Medcom GmbH (Germany); G. Sakas, Fraunhofer IGD (Germany); S. Walter, Medcom GmbH (Germany); W.-D. Groch, Univ. of Applied Sciences (Germany); U. Stilla, Technische Univ. München (Germany)

$69142 \mathrm{~V}$ Registration of standardized histological images in feature space [6914-103]

U. Bağci, L. Bai, The Univ. of Nottingham (United Kingdom)

$69142 \mathrm{~W} \quad$ A new parametric nonrigid image registration method based on Helmholtz's theorem [6914-104]

H.-Y. Hsiao, H. Chen, T.-H. Lin, C.-Y. Hsieh, M.-Y. Chu, G. Liao, The Univ. of Texas at Arlington (USA); H. Zhong, Virginia Commonwealth Univ. (USA)

$69142 X \quad 3-D$ statistical cancer atlas-based targeting of prostate biopsy using ultrasound image guidance [6914-105]

R. Narayanan, Eigen LLC (USA); D. Shen, C. Davatzikos, Univ. of Pennsylvania (USA);

E. D. Crawford, A. Barqawi, P. Werahera, Univ. of Colorado Denver (USA); D. Kumar,

J. S. Suri, Eigen LLC (USA)

$69142 Y \quad$ Optimized GPU implementation of learning-based non-rigid multi-modal registration [6914-107]

Z. Fan, Stony Brook Univ. (USA); C. Vetter, C. Guetter, D. Yu, Siemens Corporate Research (USA); R. Westermann, Technische Univ. München (Germany); A. Kaufman, Stony Brook Univ. (USA); C. XU, Siemens Corporate Research (USA)

$69142 Z$ Efficient 3D rigid-body registration of micro-MR and micro-CT trabecular bone images [6914-108]

C. S. Rajapakse, J. Magland, Univ. of Pennsylvania School of Medicine (USA); S. L. Wehrli, Children's Hospital of Philadelphia (USA); X. H. Zhang, X. S. Liu, X. E. GUo, Columbia Univ. (USA); F. W. Wehrli, Univ. of Pennsylvania School of Medicine (USA)

691430 Histopathology and MR image fusion of the prostate [6914-109]

H. H. Jo, J. Jung, Y. Jang, H. Hong, Seoul Women's Univ. (South Korea); H. J. Lee, Seoul National University Bundang Hospital (South Korea) 
691431 3D-3D alignment using particle swarm optimization [6914-110] S. Schafer, K. R. Hoffmann, P. B. Noël, C. L. Bloebaum, State Univ. of New York at Buffalo (USA)

691432 Automatic alignment of renal DCE-MRI image series for improvement of quantitative tracer kinetic studies [6914-111]

D. Zikic, Technische Univ. München (Germany); S. Sourbron, University Hospitals MunichGrosshadern (Germany); X. Feng, Technische Univ. München (Germany); H. J. Michaely, University Hospitals Munich-Grosshadern (Germany); A. Khamene, Siemens Corporate Research (USA); N. Navab, Technische Univ. München (Germany)

691433 Consistent detection of mid-sagittal planes for follow-up MR brain studies [6914-112] Y. Wang, Univ. of Florida (USA); L. Zhang, Siemens Corporate Research (USA)

691434 A rapid and robust iterative closest point algorithm for image-guided radiotherapy [6914-113]

J. Barbiere, J. Hanley, Hackensack Univ. Medical Ctr. (USA)

691435 Retinal image mosaicing using the radial distortion correction model [6914-114] S. Lee, The Univ. of lowa (USA); M. D. Abràmoff, The Univ. of lowa (USA) and Veteren's Administration Medical Ctr. (USA); J. M. Reinhardt, The Univ. of Iowa (USA)

691436 Deformation estimation and analysis for adaptive radiation therapy [6914-115] B. Wang, J. Xuan, Virginia Polytechnic Institute and State Univ. (USA); J. Q. Wu, Duke Univ. (USA); S. Zhang, Shanghai Jiao Tong Univ. (China); Y. Wang, Virginia Polytechnic Institute and State Univ. (USA)

\section{Part Three}

\section{POSTERS: SEGMENTATION}

691437 Simultaneous segmentation and motion estimation in 4D-CT data using a variational approach [6914-116]

J. Ehrhardt, A. Schmidt-Richberg, H. Handels, University Medical Ctr. Hamburg-Eppendorf (Germany)

691438 Recent advances in 3D-CSC based MR brain image segmentation [6914-117] F. Schmitt, L. Priese, Univ. Koblenz-Landau (Germany)

691439 Automatic knee cartilage delineation using inheritable segmentation [6914-120] S. P. M. Dries, V. Pekar, D. Bystrov, H. S. Heese, T. Blaffert, Philips Research Europe - Hamburg (Germany); C. Bos, A. M. C. van Muiswinkel, Philips Medical Systems (Netherlands)

6914 3A Computerized image analysis for acetic acid induced intraepithelial lesions [6914-121] W. Li, STI Medical Systems (USA); D. G. Ferris, Medical College of Georgia (USA); R. W. Lieberman, Univ. of Michigan Medical School (USA) 
6914 3B Improving 3D active appearance model segmentation of the left ventricle with Jacobian tuning [6914-122]

K. Y. E. Leung, Erasmus Medical Ctr. (Netherlands); M. van Stralen, Erasmus Medical Ctr. (Netherlands), Interuniversity Cardiology Institute of the Netherlands (Netherlands), and Leiden University Medical Ctr. (Netherlands); M. M. Voormolen, N. de Jong,

A. F. W. van der Steen, Erasmus Medical Ctr. (Netherlands) and Interuniversity Cardiology Institute of the Netherlands (Netherlands); J. H. C. Reiber, Leiden University Medical Ctr. (Netherlands); J. G. Bosch, Erasmus Medical Ctr. (Netherlands)

6914 3C Novel method for digital subtraction of tagged stool in virtual colonoscopy [6914-123] L. Guendel, M. Suehling, Siemens Healthcare Sector (Germany); H. Eckert, Siemens Corporate Technology (Germany)

6914 3D Airway segmentation by topology-driven local thresholding [6914-124] A. Szymczak, Colorado School of Mines (USA); J. Vanderhyde, IronCAD (USA)

6914 3E Improving cervical region of interest by eliminating vaginal walls and cotton-swabs for automated image analysis [6914-125]

S. Venkataraman, W. Li, STI Medical Systems (USA)

6914 3F CALM: cascading system with leaking detection mechanism for medical image segmentation [6914-126]

J. LiU, J. H. Lim, H. Li, Institute for Infocomm Research, A*STAR (Singapore)

$69143 G$ Validation of automatic landmark identification for atlas-based segmentation for radiation treatment planning of the head-and-neck region [6914-127]

C. Leavens, Univ. of Toronto (Canada); T. Vik, H. Schulz, Philips Research Europe (Germany); S. Allaire, J. Kim, L. Dawson, B. O'Sullivan, S. Breen, D. Jaffray, Univ. of Toronto (Canada); V. Pekar, Philips Research North America (Canada)

691431 Segmentation in noisy medical images using PCA model based particle filtering [6914-129] W. QU, Siemens Medical Solutions USA Inc. (USA); X. Huang, Lehigh Univ. (USA); Y. Jia, Univ. of Illinois at Chicago (USA)

6914 3K Semi-automatic detection of Gd-DTPA-saline filled capsules for colonic transit time assessment in MRI [6914-131]

C. Harrer, Technische Univ. München (Germany); S. Kirchhoff, Univ. Hospital Munich Großhadern (Germany); A. Keil, Technische Univ. München (Germany) and Univ. Hospital Munich - Innenstadt (Germany); C. Kirchhoff, T. Mussack, Univ. Hospital Munich - Innenstadt (Germany); A. Lienemann, M. Reiser, Univ. Hospital Munich - Großhadern (Germany); N. Navab, Technische Univ. München (Germany)

6914 3L A learning-based automatic spinal MRI segmentation [6914-132] X. Liu, J. Samarabandu, The Univ. of Western Ontario (Canada); G. Garvin, St. Joseph's Health Care (Canada); R. Chhem, London Health Sciences Ctr. (Canada); S. Li, GE Healthcare (Canada)

$69143 \mathrm{M}$ Reclassification of segmentation boundary base on neighboring function [6914-133] J. Chen, J. Tian, Institute of Automation (China) 
$69143 \mathrm{~N} \quad$ Effect of various binning methods and ROI sizes on the accuracy of the automatic classification system for differentiation between diffuse infiltrative lung diseases on the basis of texture features at HRCT [6914-134]

N. Kim, Asan Medical Ctr. (South Korea) and Seoul National Univ. (South Korea); J. B. Seo, Asan Medical Ctr. (South Korea); Y. S. Sung, B.-W. Park, Y. Lee, Seoul National Univ. (South Korea); S. H. Park, Y. K. Lee, Asan Medical Ctr. (South Korea); S.-H. Kang, Seoul National Univ. (South Korea)

691430 Interactive surface correction for 3D shape-based segmentation [6914-136] T. Schwarz, T. Heimann, R. Tetzlaff, A.-M. Rau, I. Wolf, H.-P. Meinzer, German Cancer Research Ctr. (Germany)

6914 3P An approach to segment lung pleura from CT data with high precision [6914-137] E. Angelats, RWTH Aachen Univ. (Germany); K. Chaisaowong, RWTH Aachen Univ. (Germany) and King Mongkut's Institute of Technology (Thailand); A. Knepper, RWTH Aachen Univ. (Germany); T. Kraus, Univ. Hospital Aachen (Germany); T. Aach, RWTH Aachen Univ. (Germany)

$69143 Q \quad$ Boundary-precise segmentation of nucleus and plasma of leukocytes [6914-138] T. Zerfaß, T. Rehn, T. Wittenberg, Fraunhofer Institute for Integrated Circuits (Germany)

6914 3R Fully automated segmentation of carotid and vertebral arteries from contrast-enhanced CTA [6914-139]

O. Cuisenaire, Philips Healthcare (France); S. Virmani, M. E. Olszewski, Philips Healthcare (USA); R. Ardon, Philips Healthcare (France)

691435 Simultaneous detection of multiple elastic surfaces with application to tumor segmentation in CT images [6914-140]

K. Li, Carnegie Mellon Univ. (USA); M.-P. Jolly, Siemens Corporate Research (USA)

6914 3T An efficient topology adaptation system for parametric active contour segmentation of 3D images [6914-141]

J. Abhau, Institut für Informatik (Austria); O. Scherzer, Institut für Informatik (Austria) and IMCC Linz (Austria)

$69143 \mathrm{U}$ Time-dependent joint probability speed function for level-set segmentation of rat brain slices [6914-142]

C. Palm, Univ. College London (United Kingdom) and Research Ctr. Jülich (Germany); U. Pietrzyk, Research Ctr. Jülich (Germany) and Univ. Wuppertal (Germany)

6914 3V Multi-phase image segmentation using level sets [6914-143]

P. Zhilkin, M. Alexander, Institute for Biodiagnositcs (Canada)

6914 3W Bidirectional segmentation of prostate capsule from ultrasound volumes: an improved strategy [6914-144]

L. Wei, R. Narayanan, D. Kumar, Eigen LLC (USA); A. Fenster, Imaging Research Labs. (Canada); A. Barqawi, P. Werahera, E. D. Crawford, Univ. of Colorado Hospital (USA); J. S. Suri, Imaging Research Labs. (Canada) 
$69143 \mathrm{X} \quad$ Robust segmentation using kernel and spatial based fuzzy c-means methods on breast x-ray images [6914-145]

X. Sun, D. Goldgof, Univ. of South Florida (USA); W. Land, Binghamton Univ. (USA)

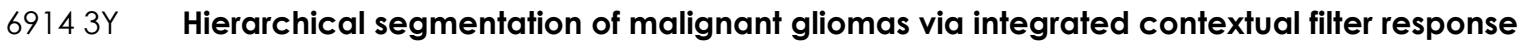
[6914-147]

S. Dube, J. J. Corso, A. Yuille, T. F. Cloughesy, S. El-Saden, U. Sinha, Univ. of California, Los Angeles (USA)

$69143 Z$ Local control of speed function in level set segmentation using interactive interface for CT images [6914-148]

S. Tate, N. Tsumura, T. Nakaguchi, Y. Miyake, Chiba Univ. (Japan)

691440 Automated retinal layer segmentation in OCT images using spatially variant filtering [6914-149]

A. M. Bagci, R. Ansari, M. Shahidi, Univ. of Illinois at Chicago (USA)

$691441 \quad$ Neuronal nuclei localization in 3D using level set and watershed segmentation from laser scanning microscopy images [6914-150]

Y. Zhu, Syracuse Univ. (USA); E. Olson, SUNY Upstate Medical Univ. (USA); A. Subramanian, Syracuse Univ. (USA); D. Feiglin, SUNY Upstate Medical Univ. (USA); P. K. Varshney, Syracuse Univ. (USA); A. Krol, SUNY Upstate Medical Univ. (USA)

691442 Prostate segmentation on pelvic CT images using a genetic algorithm [6914-151] P. Ghosh, Portland State Univ. (USA); M. Mitchell, Portland State Univ. (USA) and Santa Fe Institute (USA)

691443 Robust segmentation of tubular structures in medical images [6914-152] R. Fahmi, Siemens Medical Solutions (USA) and Univ. of Louisville (USA); A. Jerebko, M. Wolf, Siemens Medical Solutions (USA); A. A. Farag, Univ. of Louisville (USA)

691444 Segmentation of large periapical lesions toward dental computer-aided diagnosis in cone-beam CT scans (Honorable Mention Poster Award) [6914-153]

S. Rysavy, A. Flores, San Francisco State Univ. (USA); R. Enciso, Univ. of Southern California (USA); K. Okada, San Francisco State Univ. (USA)

691445 Segmentation of sonographic breast lesions: fuzzy cell-competition algorithm and bias field reduction [6914-154]

C.-Y. Lee, C.-C. Hsieh, C.-M. Chen, National Taiwan Univ. (Taiwan)

691447 Three-dimensional segmentation of bones from CT and MRI using fast level sets [6914-156] J. Krátký, J. Kybic, Czech Technical Univ. (Czech Republic)

691448 3-D segmentation of articular cartilages by graph cuts using knee MR images from osteoarthritis initiative [6914-157]

H. Shim, Univ. of Pittsburgh (USA); S. Lee, B. Kim, Seoul National Univ. (South Korea); C. Tao,

S. Chang, Univ. of Pittsburgh (USA); I. D. Yun, Hankuk Univ. of Foreign Studies (South Korea);

S. U. Lee, Seoul National Univ. (South Korea); K. Kwoh, K. Bae, Univ. of Pittsburgh (USA) 
691449 Segmentation and volumetric measurement of renal cysts and parenchyma from MR images of polycystic kidneys using multi-spectral analysis method [6914-158]

K. T. Bae, Univ. of Pittsburgh (USA); P. K. Commean, B. S. Brunsden, Washington Univ. in St. Louis (USA); D. A. Baumgarten, Emory Univ. School of Medicine (USA); B. F. King, Jr., Mayo Foundation (USA); L. H. Wetzel, Univ. of Kansas Medical Ctr. (USA); P. J. Kenney, Univ. of Alabama at Birmingham (USA); A. B. Chapman, Emory Univ. School of Medicine (USA); V. E. Torres, Mayo Foundation (USA); J. J. Grantham, Univ. of Kansas Medical Ctr. (USA); L. M. Guay-Woodford, Univ. of Alabama at Birmingham (USA); C. Tao, Univ. of Pittsburgh (USA); J. P. Miller, Washington Univ. in St. Louis (USA); C. M. Meyers, National Institutes of Health (USA); W. M. Bennett, Northwest Renal Clinic (USA)

6914 4A Semi-automated segmentation of the prostate gland boundary in ultrasound images using a machine learning approach [6914-159]

K. Diaz, Pontificia Univ. Católica del Perú (Peru); B. Castaneda, Pontificia Univ. Católica del Perú (Peru) and Univ. of Rochester (USA)

6914 4B Multiscale support vector clustering [6914-160]

M. S. Hansen, Technical Univ. of Denmark (Denmark); D. A. Holm, Technical Univ. of Denmark (Denmark) and Danish Research Ctr. for Magnetic Resonance, Copenhagen Univ. Hospital (Denmark); K. Sjöstrand, Technical Univ. of Denmark (Denmark); C. D. Ley, Institute for Molecular Pathology (Denmark); I. J. Rowland, Danish Research Ctr. for Magnetic Resonance, Copenhagen Univ. Hospital (Denmark); R. Larsen, Technical Univ. of Denmark (Denmark)

6914 4C Fast approximate surface evolution in arbitrary dimension [6914-161] J. Malcolm, Georgia Institute of Technology (USA); Y. Rathi, Brigham and Women's Hospital (USA); A. Yezzi, A. Tannenbaum, Georgia Institute of Technology (USA)

6914 4D An accurate segmentation method for volumetry of brain tumor in 3D MRI [6914-162] J. Wang, Q. Li, Duke Univ. (USA); T. Hirai, S. Katsuragawa, Kumamoto Univ. (Japan); F. Li, K. Doi, The Univ. of Chicago (USA)

$69144 \mathrm{E}$ Automated segmentation of middle hepatic vein in non-contrast $\mathrm{x}$-ray $\mathrm{CT}$ images based on an atlas-driven approach [6914-163]

T. Kitagawa, X. Zhou, T. Hara, H. Fujita, Gifu Univ. Graduate School of Medicine (Japan);

R. Yokoyama, Gifu Univ. School of Medicine (Japan); H. Kondo, Gifu Univ. Hospital (Japan); M. Kanematsu, Gifu Univ. School of Medicine (Japan); H. Hoshi, Gifu Univ. Graduate School of Medicine (Japan)

$69144 \mathrm{~F}$ A deformable model-based minimal path segmentation method for kidney MR images [6914-164]

K. Li, B. Fei, Case Western Reserve Univ. (USA)

6914 4G Automated lung tumor detection and quantification for respiratory gated PET/CT images [6914-165]

J. Wang, M. del Valle, Florida International Univ. (USA); J. Franquiz, Radiological Physics of South Florida, Inc. (USA); A. McGoron, Florida International Univ. (USA) 
$69144 \mathrm{H} \quad$ Efficient curvature estimations for real-time $(25 \mathrm{~Hz})$ segmentation of volumetric ultrasound data [6914-166]

C. R. Wagner, Cybernet Systems Corp. (USA); D. P. Perrin, Harvard Medical School (USA)

69144 Semi-automatic segmentation and modeling of the cervical spinal cord for volume quantification in multiple sclerosis patients from magnetic resonance images [6914-167] P. Sonkova, I. E. Evangelou, A. Gallo, F. K. Cantor, J. Ohayon, H. F. McFarland, F. Bagnato, National Institutes of Health (USA)

$69144 \mathrm{~J}$ Integrating local voxel classification and global shape models for medical image segmentation [6914-168]

E. M. van Rikxoort, University Medical Ctr. Utrecht (Netherlands); M. de Bruijne, Univ. of Copenhagen (Denmark) and Erasmus Medical Ctr. (Netherlands); B. van Ginneken, University Medical Ctr. Utrecht (Netherlands)

$69144 \mathrm{~K}$ Lung lobe and segmental lobe extraction from 3D chest CT datasets based on figure decomposition and Voronoi division [6914-169]

K. Mori, Y. Nakada, T. Kitasaka, Y. Suenaga, Nagoya Univ. (Japan); H. Takabatake, Sapporo-Minami-sanjo Hospital (Japan); M. Mori, Sapporo-Kosei General Hospital (Japan); H. Natori, Keiwakai Nishioka Hospital (Japan)

POSTERS: SHAPE

$69144 \mathrm{M}$ A novel approach to fracture-risk-assessment in osteoporosis by ROI-oriented application of the Minkowski-functionals to dual x-ray absorptiometry scans of the hip [6914-171] H. F. Boehm, A. Panteleon, T. Vogel, D. Burklein, M. Reiser, Univ. of Munich (Germany)

$69144 \mathrm{~N}$ Reconstructing liver shape and position from MR image slices using an active shape model [6914-172]

M. Fenchel, Siemens Medical Solutions (Germany) and Eberhard-Karls-Univ. (Germany); S. Thesen, Siemens Medical Solutions (Germany); A. Schilling, Eberhard-Karls-Univ. (Germany)

691440 Tracheal stent prediction using statistical deformable models of tubular shapes [6914-173] R. Pinho, T. Huysmans, Univ. of Antwerp (Belgium); W. Vos, University Hospital of Antwerp (Belgium); J. Sijbers, Univ. of Antwerp (Belgium)

6914 4P Vertebral classification using localized pathology-related shape model [6914-174] R. Zewail, A. Elsafi, N. Durdle, Univ. of Alberta (Canada)

$69144 Q \quad$ Local curvature scale: a new concept of shape description [6914-175] S. Rueda, The Univ. of Nottingham (United Kingdom); J. K. Udupa, Univ. of Pennsylvania (USA); L. Bai, The Univ. of Nottingham (United Kingdom)

$69144 \mathrm{R}$ Conditional-mean initialization using neighboring objects in deformable model segmentation (Honorable Mention Poster Award) [6914-176] J.-Y. Jeong, J. V. Stough, J. S. Marron, S. M. Pizer, Univ. of North Carolina, Chapel Hill (USA) 
6914 4S A multi-modal prostate segmentation scheme by combining spectral clustering and active shape models [6914-177]

R. Toth, P. Tiwari, Rutgers Univ. (USA); M. Rosen, Univ. of Pennsylvania (USA); A. Kalyanpur, Teleradiology Solutions (India); S. Pungavkar, Dr. Balabhai Nanavati Hospital (India);

A. Madabhushi, Rutgers Univ. (USA)

$69144 \mathrm{~T}$ Comparison of statistical shape models built on correspondence probabilities and one-toone correspondences [6914-178]

H. Hufnagel, University Medical Ctr. Hamburg-Eppendorf (Germany); X. Pennec, INRIA Sophia Antipolis (France); J. Ehrhardt, University Medical Ctr. Hamburg-Eppendorf (Germany); N. Ayache, INRIA Sophia Antipolis (France); H. Handels, University Medical Ctr. Hamburg-Eppendorf (Germany)

POSTERS: TEXTURE

$69144 \mathrm{U}$ Studying the effect of noise on the performance of 2D and 3D texture measures for quantifying the trabecular bone structure as obtained with high resolution MR imaging at 3 tesla [6914-179]

R. Monetti, Max-Planck-Institut für extraterrestrische Physik (Germany); J. Bauer, D. Mueller, E. J. Rummeny, Technische Univ. München (Germany); T. M. Link, S. Majumdar, Univ. of California, San Francisco (USA); M. Matsuura, Ludwig-Maximilians-Univ. (Germany);

F. Eckstein, Paracelsus Medical Univ. (Austria); I. Sidorenko, C. W. Raeth, Max-Planck-Institut für extraterrestrische Physik (Germany)

$69144 \mathrm{~V}$ Comparison and combination of scaling index method and Minkowski functionals in the analysis of high resolution magnetic resonance images of the distal radius in vitro [6914-180]

I. N. Sidorenko, Max-Planck-Institut für extraterrestrische Physik (Germany); J. Baver, Technische Univ. Muenchen (Germany); R. Monetti, Max-Planck-Institut für extraterrestrische Physik (Germany); D. Mueller, E. J. Rummeny, Technische Univ. Muenchen (Germany); F. Eckstein, Paracelsus Medical Private Univ. (Austria); C. W. Raeth, Max-PlanckInstitut für extraterrestrische Physik (Germany)

\section{POSTERS: VALIDATION}

$69144 \mathrm{~W}$ Semi-synthetic digital phantoms incorporating natural structured noise and boundary inhomogeneities [6914-181]

S. Tan, M. M. Ward, National Institutes of Health (USA)

6914 4X Evaluation of accuracy in partial volume analysis of small objects [6914-182]

J. Rexilius, H.-O. Peitgen, MeVis Research (Germany)

$69144 \mathrm{Y}$ A software assistant for the design of realistic software phantoms [6914-183]

J. Rexilius, O. Konrad, H.-O. Peitgen, MeVis Research (Germany)

Author Index 


\title{
Conference Committee
}

\author{
Symposium Chairs
}

Milan Sonka, The University of lowa (USA)

Armando Manduca, Mayo Clinic (USA)

\section{Conference Chairs}

Joseph M. Reinhardt, The University of lowa (USA)

Josien P. W. Pluim, Universitair Medisch Centrum Utrecht (Netherlands)

Program Committee

Mostafa Analoui, Pfizer Inc. (USA)

Kyongtae Ty Bae, University of Pittsburgh (USA)

Christian Barillot, IRISA (France)

Benoit M. Dawant, Vanderbilt University (USA)

Aaron Fenster, Robarts Research Institute (Canada)

Alejandro F. Frangi, Universitat Pompeu Fabra (Spain)

James C. Gee, University of Pennsylvania (USA)

Guido Gerig, University of Utah (USA)

David R. Haynor, University of Washington (USA)

Tianhu Lei, University of Pennsylvania (USA)

Boudewijn P. F. Lelieveldt, Leids Universitair Medisch Centrum (Netherlands)

Bostjan Likar, Univerza v Ljubljani (Slovenia)

Murray H. Loew, George Washington University (USA)

Anthony J. Maeder, CSIRO Information and Communication Technologies Centre (Australia)

Frederik Maes, Katholieke Universiteit Leuven (Belgium)

Sunanda D. Mitra, Texas Tech University (USA)

Kensaku Mori, Nagoya University (Japan)

Sébastien Ourselin, University College London (United Kingdom)

Daniel Rueckert, Imperial College London (United Kingdom)

Punam K. Saha, The University of lowa (USA)

Julia A. Schnabel, St. Hilda's College (United Kingdom) and University of Oxford (United Kingdom)

Colin Studholme, University of California, San Francisco (USA)

Philippe Thévenaz, Ecole Polytechnique Fédérale de Lausanne

(Switzerland)

Jayaram K. Udupa, University of Pennsylvania (USA)

Bram van Ginneken, Universitair Medisch Centrum Utrecht

(Netherlands)

Andreas Wahle, The University of lowa (USA) 
Session Chairs

1 Segmentation I: Methodology

Guido Gerig, University of Utah (USA)

2 Atlases and Population Studies

Anthony J. Maeder, CSIRO Information and Communication Technologies Centre (Australia)

3 Registration I: Applications

Colin Studholme, University of California, San Francisco (USA)

$4 \quad$ Neurological Applications

James C. Gee, University of Pennsylvania (USA)

5 Classification and Pattern Recognition

Punam K. Saha, The University of lowa (USA)

6 Registration II: Methodology

Frederik Maes, Katholieke Universiteit Leuven (Belgium)

7 Cardiovascular Applications

Andreas Wahle, The University of lowa (USA)

$8 \quad$ Image Restoration and Enhancement

David R. Haynor, University of Washington (USA)

9 Keynote and Liver Applications

Kensaku Mori, Nagoya University (Japan)

10 Pulmonary Applications

Kyongtae Ty Bae, University of Pittsburgh (USA)

11 Segmentation II: Applications

Boudewijn P. Lelieveldt, Leids Universitair Medisch Centrum

(Netherlands) 


\section{Introduction}

These proceedings contain the papers presented at the Image Processing conference of the 2008 SPIE Medical Imaging Symposium, held 16-21 February at the Town and Country Hotel in San Diego, California. A total of 120 posters and 63 oral presentations were selected from 264 submissions. The high quality of the submissions made the paper selection process extremely difficult, and in the end we were limited by the available timeslots for oral presentations and space for poster presentations. The acceptance rate this year was $69 \%$. The total number of submissions increased by $15 \%$ compared to 2007 . Of the 183 accepted papers, 170 were presented at the meeting and are included in these proceedings.

The first day of the conference ended with the evening workshop entitled "DICOM Working Group 23 and Application Hosting: What They Mean for Research in Medical Image Analysis," organized and chaired by Dr. David Haynor, of the University of Washington. Dr. Haynor opened the workshop by describing how difficult it can be to bring a new research image processing application into a clinical radiology environment; the reading workstations are typically locked down so that no new software can be added, necessitating that a new computer be added to the reading room to deploy a new application. Four speakers from academia, industry, and government discussed different aspects of application hosting. Dr. Lawrence Tarbox, of Washington University in St. Louis, described the work done by DICOM Working Group 23 to define a standard application hosting framework that provides a "plug-in" interface so that new applications could be loaded into a workstation at runtime, and could run in a safe, protected environment. He described the development of the eXtensible Imaging Platform (XIP), an open-source environment for rapidly developing medical imaging applications. Dr. Gianluca Paladini, of Siemens Corporate Research, discussed the Siemens OpenXIP environment for rapid application development. OpenXIP provides easy access to image processing and display libraries such as ITK and VTK. Mr. Chris Wood, of Clario Medical Imaging, described a Clario application hosting environment, and discussed how researchers might work with Clario to distribute and support their image processing applications. Dr. Laurence Clarke, of the National Cancer Institute $(\mathrm{NCl})$ at the $\mathrm{NIH}$, discussed the NCl's interest in application hosting, portable plugins, and, more generally, open-source software. After the four speakers delivered their prepared remarks, there was an interesting panel discussion and questionand-answer session. Various practical considerations of using application hosting and plug-ins in a clinical environment, such as FDA approval, liability, and royalties were discussed. After the workshop, the five panelists and many audience members moved to a conference room for a hands-on session with the XIP environments. 
Our keynote speaker this year was Dr. James Duncan, from Yale University. Dr. Duncan is well known in the medical imaging community for his work in many areas of medical image processing, and for his founding and leadership of the Image Processing and Analysis Group (IPAG) at Yale University. Dr. Duncan's keynote talk was entitled "Model-based biomedical image analysis." He described the challenges associated with accurately and reproducibly extracting useful quantitative information from medical images, including image acquisition limitations, the variability of normal human anatomy and physiology, and the presence of disease or other abnormal conditions. Dr. Duncan argued that robust and reproducible image analysis solutions combine both imagederived information and model-based information. He discussed research performed at IPAG to use shape, geometry, and biomechanical models to guide image analysis and to constrain the range of plausible solutions in the presence of uncertainty, and described key applications of these concepts in the areas of cardiac and neurological image analysis.

The image processing posters were displayed on Sunday and Monday this year. At the Monday night poster reception, one cum laude poster and three honorable mention posters were recognized as the winners of the student poster competition. The award-winning poster papers are marked in these proceedings.

Many people contributed time and energy toward making the conference a success. We would like to thank the members of the Image Processing Conference program committee for their efforts before and during the meeting in reviewing paper submissions, chairing sessions, and judging posters. We would also like to thank the staff at SPIE for their work in organizing the meeting and publishing the proceedings. And, of course, we are grateful to all of the authors, for they are the ones that produced and presented the exciting research that makes these proceedings possible. 


\section{In Memoriam}

\section{Sam Dwyer}

1932-2008

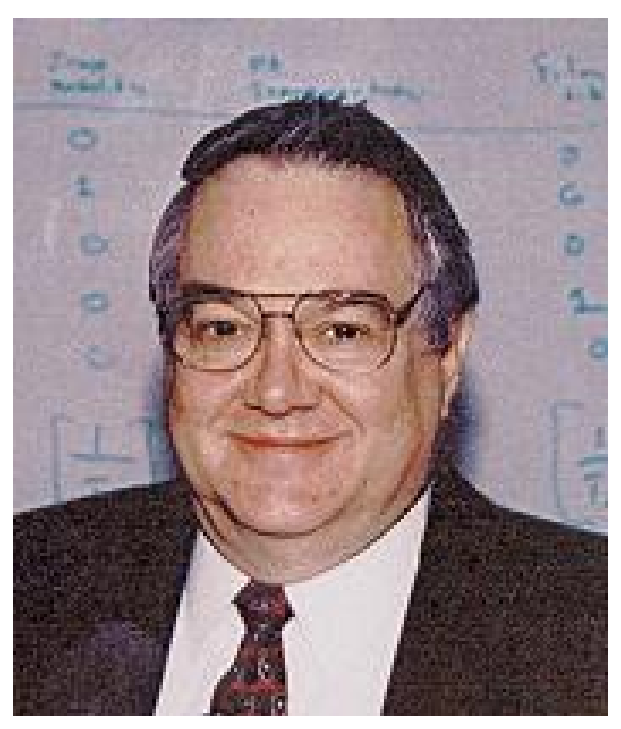

\section{Inspirational planner for SPIE Medical Imaging symposia and chair of 15 conferences from 1983 to 1996}

Sam Dwyer was a leading light in the swift growth of the field of medical imaging. At this 2008 Medical Imaging symposium, several colleagues expressed their appreciation of his professional achievements and the legacy of his contributions.

Andre Duerinckx recalls Sam's first big step into the limelight. "Sam chaired the Second International PACS meeting sponsored by SPIE in 1983. He stepped in when my professional direction shifted and I could not continue. Over the following years Sam became a leading force in the development of this new science and technology as the conferences developed and grew."

"He had an ability to predict important technology trends in medical imaging," says Steven Horii, a long-time SPIE Medical Imaging contributor and past conference chair.

Another past Medical Imaging conference chair with years of experience, Roger Schneider elaborates, "The growth of the initial conferences was phenomenal. They quickly expanded and outgrew several facilities. Topic areas increased. For example, we had been dealing with perception in sessions on image statistics. Sam first suggested that the field of perception theory and experiment was expanding so rapidly that it deserved its own conference. We also added conferences on image processing hardware, display, functional imaging, and special topics in ultrasound transducers."

"Sam was a true pioneer in our field," continues Schneider. "He was one of the first to envision the impact of digital technology on the storage, retrieval, communication, and 
display of medical images, and one of the most active early explorers of the possibilities. He led the PACS conference into the merger with SPIE's image science conference to form the current Medical Imaging Symposium which many consider to be the premiere technical program on medical imaging in the world."

Murray Loew, another past conference chair, adds his observation, "Sam's academic, industrial, and clinical experience provided the perspective that enabled him to set priorities and give advice that helped many of us to make our own contributions. We all benefited greatly from his insights and his practical approach."

Schneider agrees. "Through all, Sam was an excellent partner and leader, calm, gentle yet persuasive, always congenially nudging everyone toward a better future. His ambitions were not for himself, but for the mutual enterprise. He was a very enthusiastic mentor and supporter of students and researchers new to the field without any concern for the possibility that their work might compete with his and was himself a superbly competent contributor."

An example of this is illustrated by John Strauss." Already well accomplished at the time of our first meeting, Sam made the time to take an eager but inexperienced student under his wing. I was not an 'assigned' grad student or research assistant from the University to which he had an obligation. I was a product manager from a vendorpartner. Over the years, from answering technical questions in an understandable way, to providing unassuming career guidance, as well as a sympathetic ear to the challenges of fatherhood or life's many challenges and struggles, Sam was always there for me."

"There are many things about Sam Dwyer that I recall with great fondness, like his always friendly and enthusiastic manner. His advice led me to my years of satisfying work on the ACR-NEMA Committee," adds Horii.

All agree that Sam had many other admirable traits. His wit and sense of humor were legendary, as one of Schneider's favorite memories illustrates. "At the opening of one conference Sam announced, from the podium, that it was the birthday of an important attendee. He said SPIE had requested that the Blue Angels do a flyover-but they already had something scheduled. As a substitute birthday recognition, Sam suggested we take our morning coffee break out on the terrace and watch the landscaping crew circle the flagpole on their riding lawnmowers."

Strauss describes the footprint left by Sam, "While Sam left a legacy through his professional accomplishments, perhaps more lasting is the heritage of leadership he has left behind. He felt it his obligation to pass on his knowledge and wisdom to the next generation, and I was blessed as a recipient. I have and will continue to honor Sam by sharing with those that come after me."

Sam Dwyer was a person of rare quality who will be sorely missed. His name has a permanent place in the annals of medical imaging, and his contributions continue in the flourishing growth of knowledge presented and discussed in the annual Medical Imaging conferences. 